\title{
Towards a dynamic actuator model for a hexapod robot
}

\author{
Dave McMordie, Chris Prahacs and Martin Buehler \\ mcmordie|cprahacs|buehler@cim.mcgill.ca \\ Ambulatory Robotics Laboratory, http:/ / www.mcgill.cim.ca/ arlweb \\ Centre for Intelligent Machines, McGill University, Montreal, CANADA
}

\begin{abstract}
We describe a model predicting the output torque of the battery-amplifier-actuator-gear combination used on the hexapod robot RHex, based on requested PWM (PulseWidth-Modulation) duty cycle to the amplifier, battery voltage, and motor speed. The model is broken into independent components, each experimentally validated: power source (battery), motor amplifier, motor, and (planetary) gear. The resulting aggregate model shows $<6 \%$ Full Scale RMS error in predicting output torque in the first quadrant of operation (positive torques). Understanding the key ingredients and the attainable accuracies of torque production models in our commonly used battery-amplifier-actuator-gear combinations is critical for mobile robots, in order to minimize sensing, and thus space, size, weight, power consumption, failure rate, and cost of mobile robots.
\end{abstract}

Keywords- DC permanent magnet brush motor, $P W M$, planetary gear, current, voltage, model, hexapod, robot

\section{Introduction}

RHex is a simple and highly mobile hexapod robot [4]driven by six Maxon RE 118751 brush-type $20 \mathrm{~W}$ DC motors [3], combined with Maxon 114473 33:1 planetary gears, each placed at the hip of a compliant fibreglass leg. RHex's suite of dynamic behaviours includes running speeds up to $2.4 \mathrm{~m} / \mathrm{s}$ [5], climbing up to 30 degree inclines, bounding [6], pronking [7][8][9] and stair climbing [10][11][12]. Currently, each behaviour exploits proportional-derivative control of the legs to track position and velocity trajectories generated by a clock-driven state machine. While this paradigm has resulted in impressive open-loop behaviours, precise control of leg torques may enable enhanced stability and efficiency in certain behaviours via online feedback control.

Torque control for each leg actuator may be achieved in one of three ways: (1) via feedback from a torque cell placed between the gear output shaft and the leg attachment; (2) via current sensing in the motor amplifier and (3) via estimation based on motor speed and amplifier duty cycle. Since solutions (1) and (2) require additional design complexity and expense due to sensor integration, it is natural to attempt (3) first.

To this end we present a simple model for the relationship between motor speed, amplifier duty factor and output torque, based on a collection of models of each component of the actuator system.

\section{Modelling Approach}

Physics-based analytic models have the advantage that they provide modular, provable hypotheses about the operation of the actuator, which can lead both to improved model accuracy, and insights into how to improve the actuator. What's more, analytic models are typically much lower-dimension than automatically generated purely numerical or computational models, providing simpler implementation in software. In this section, we propose simple physics-based models for each system component, which will then be evaluated experimentally in the subsequent section.

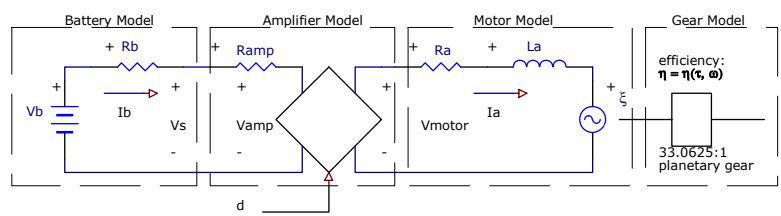

Figure 1: Compound actuator model

A single battery-amplifier-motor combination may be represented by above electrical circuit. To obtain the complete model for motor torque as a function of motor speed and duty cycle, the above circuit was broken into the three simpler models shown above, each of which was fit and validated with experimental data.

\section{Battery Model}

The battery model shown above is representative of the actuator system found in the robot. This simple internal resistance model has been used during testing and shows that the battery has the behaviour of a Thévenin-equivalent voltage source for short time intervals during discharge. However, this model cannot be used to reliably predict battery current based on voltage (or vice-versa) since the internal resistance of the battery appears to change over the course of discharge.

For the purpose of the aggregate model, battery internal resistance and internal voltage are ignored because circuitry onboard RHex measures $V_{s}$ and $I_{b}$, removing the need to estimate $V_{s}$. The foregoing argument suggests that models generated with a fixed supply voltage will generalize to variable battery voltages in the robot, so long as the battery terminal voltage can be measured and used in the model. While an estimate of $I_{b}$ is currently available on RHex, it is not necessarily desirable to depend on this 
measurement, and hence it is substituted for in the derivation that follows.

Amplifier Model

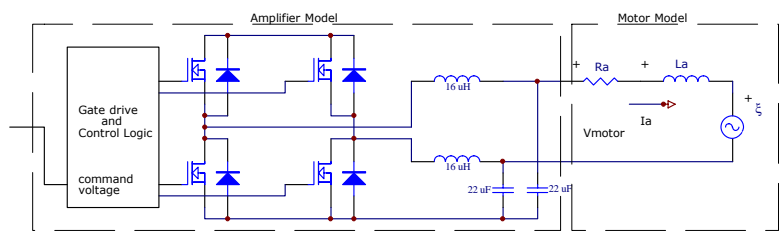

Figure 2: Detail of amplifier and motor

Figure 2 shows the electrical connection of the PWM amplifier (Apex Microtechnology SA60 [1]) and motor. Normally the PWM amplifier would be modelled together with the motor, as the winding inductance of the motor is a necessary part of the step down converter circuit, filtering out the PWM carrier frequency to produce a smooth voltage output. However, in this application a second-order LC lowpass filter with roll-off frequency $8.4 \mathrm{kHz}$ strongly attenuates the $100 \mathrm{kHz}$ PWM carrier component, leaving only a smooth voltage waveform proportional to the modulating signal, $\mathrm{d}$, (eq 1) and supply voltage at the motor terminals. As a result of this filter, the PWM switching waveform is completely removed from the output, and the system may be modelled as an ideal transformer whose output voltage is governed by the buck converter equation [2]:

$$
\mathrm{V}_{\text {motor }}=\mathrm{d} \cdot \mathrm{V}_{\text {amp }}, \mathrm{d} \in[-1,1]
$$

Assuming no power loss across the ideal transformer, we can write $I_{b} \cdot V_{\text {amp }}=V_{\text {motor }} \cdot I_{a}$ or $V_{\text {motor }}=\mathrm{d} \cdot V_{\text {amp }}$, where ' $d$ ' is the duty factor. Using this equality, an expression may be derived for the motor terminal voltage in terms of the source voltage, motor terminal current and duty cycle:

$$
\begin{aligned}
& \mathrm{V}_{\text {motor }}=\mathrm{d} \cdot \mathrm{V}_{\mathrm{amp}}=\mathrm{d} \cdot\left(\mathrm{V}_{\mathrm{s}}-\mathrm{I}_{\mathrm{b}} \cdot \mathrm{R}_{\text {amp }}\right) \\
& \mathrm{V}_{\text {motor }}=\mathrm{d} \cdot\left(\mathrm{V}_{\mathrm{s}}-\mathrm{d} \cdot \mathrm{I}_{\mathrm{a}} \cdot \mathrm{R}_{\text {amp }}\right)
\end{aligned}
$$

\section{Motor Model}

Perhaps the simplest model for a brush-type DC permanent magnet motor is the linear circuit model, derived by means of Kirchoff's voltage law:

$$
I_{a}=\frac{V_{\text {motor }}-\xi-L_{a} \frac{d_{a}}{d t}}{R_{a}}
$$

In addition, the back emf, $\xi$, and output torque, $\tau$, as a function of armature current are given by:

$$
\begin{aligned}
& \xi=\mathrm{K}_{\mathrm{s}} \omega \\
& \tau_{\text {motor }}=\mathrm{K}_{\mathrm{T}} \mathrm{I}_{\mathrm{a}}
\end{aligned}
$$

Eq. 3 is further simplified by neglecting $\mathrm{L}_{a} \frac{\mathrm{dI}_{\mathrm{a}}}{\mathrm{dt}}$ and substituting for back emf, a simplification that relies on the small winding inductance $(120 \mu \mathrm{H})$, and on the low frequency command signal:

$$
\mathrm{I}_{\mathrm{a}}=\frac{\mathrm{V}_{\text {motor }}-\mathrm{K}_{\mathrm{s}} \omega}{\mathrm{R}_{\mathrm{a}}}
$$

By combining the above equations, an expression for the motor terminal current in terms of source voltage, motor speed and duty cycle is found:

$$
I_{a}=\frac{d \cdot V_{s}-K_{s} \omega}{R_{a}+d^{2} R_{a m p}}
$$

Output torque, based on armature current, is simply modeled as:

$$
\tau_{\text {motor }}=\mathrm{K}_{\mathrm{t}} \mathrm{I}_{\mathrm{a}}=\mathrm{K}_{\mathrm{t}} \frac{\mathrm{d} \cdot \mathrm{V}_{\mathrm{s}}-\mathrm{K}_{\mathrm{s}} \omega}{\mathrm{R}_{\mathrm{a}}+\mathrm{d}^{2} \mathrm{R}_{\mathrm{amp}}}
$$

\section{Gear Model}

Though more sophisticated models exist for planetary gears, we begin with the simplest model likely to produce accurate prediction of torque:

$$
\begin{aligned}
& \tau_{\text {load }}=\mathrm{N} \cdot \eta \cdot \tau_{\text {motor }} \\
& \omega_{\text {load }}=\frac{\omega_{\text {motor }}}{\mathrm{N}}
\end{aligned}
$$

where $\mathrm{N}$ is the gear ratio and $\eta$ is the gear efficiency.

Based on eq's. 1-6, the complete model predicting output torque as a function of command signal and motor speed is given by:

$$
\tau_{\text {motor }}=\mathrm{N} \cdot \eta \cdot \mathrm{K}_{\mathrm{t}} \frac{\mathrm{d} \cdot \mathrm{V}_{\mathrm{s}}-\mathrm{K}_{\mathrm{s}} \omega}{\mathrm{R}_{\mathrm{a}}+\mathrm{d}^{2} \mathrm{R}_{\mathrm{amp}}}
$$

Though this model undoubtedly neglects certain effects at each stage, it is simple, requires little sensing, and may be easily implemented in software. Before proceeding with more detailed modeling it is instructive to examine the performance of this simple model.

\section{Dynamometer Overview}

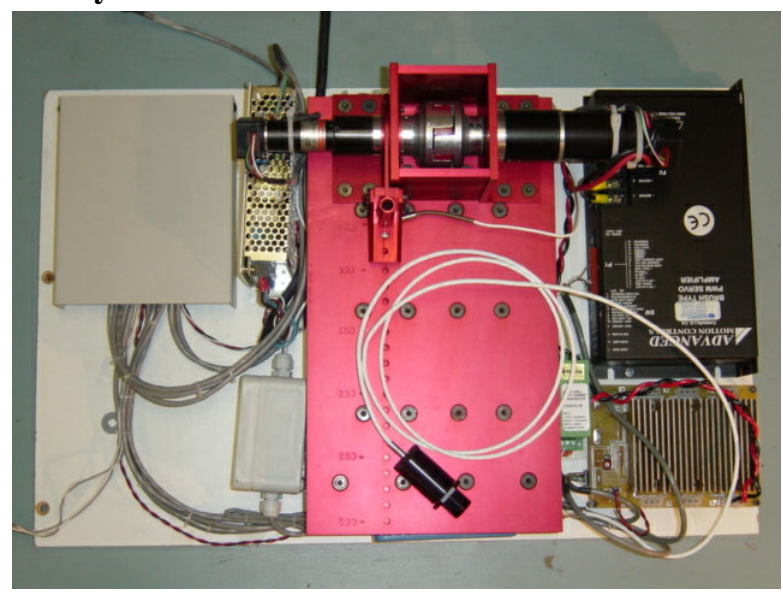

Figure 3: Dynamometer test setup

To facilitate testing of motors under dynamically realistic conditions, the authors constructed a dynamometer consisting of the actuator system under test, torque and speed measurement apparatus, and a load motor capable of torques and speeds well beyond the range of the device under test (DUT). The actuator system was fully instrumented for measurement of supply voltage and current, motor terminal voltage, motor current and motor case temperature using a National Instruments PCI-6036E 16-bit data acquisition card. 
Using National Instruments LabVIEW ${ }^{\mathrm{TM}}$ a program was developed to perform measurements, proportional-integral (PI) control of load motor speed and control of the device-under-test command signal at a rate of $50 \mathrm{~Hz}$. Accuracies for each measurement device used in this set-up are given in Table 1.

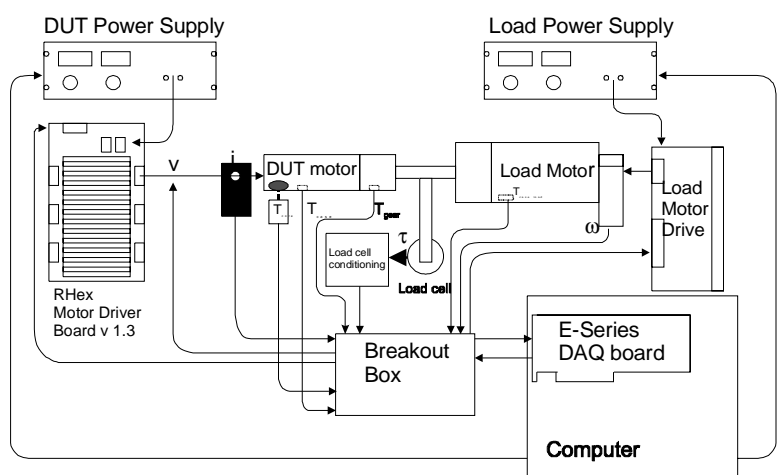

Figure 4: Dynamometer electrical test set-up

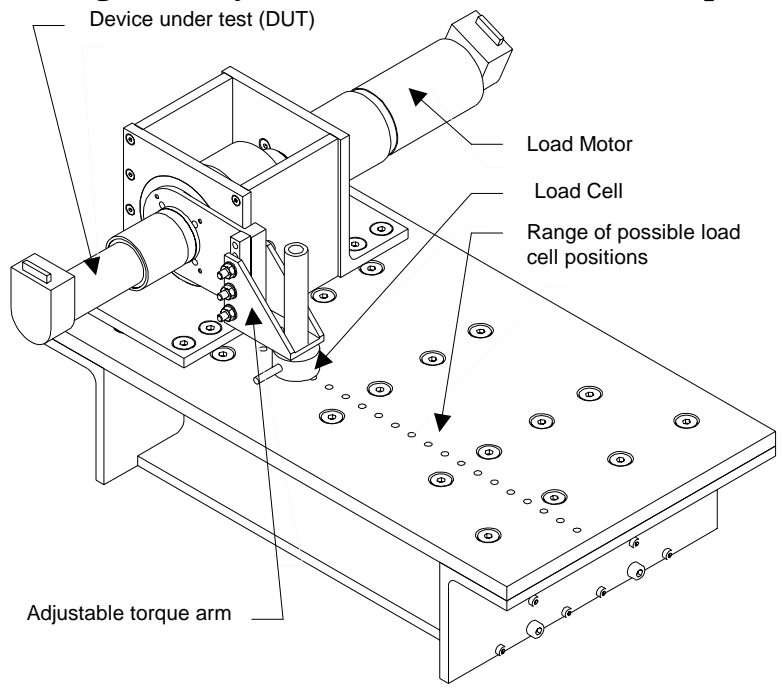

Figure 5: Dynamometer mechanical test setup

The physical design of the dynamometer provides a rigid connection between the motors and load cell. The load cell is attached to the DUT by means of a variable length torque arm. The torque arm is attached directly to the gearhead, providing a direct and rigid link with which to measure the reaction force at the load cell. The DUT and torque arm are mounted on a bearing, to isolate the load cell as the sole reaction point for motor output torque. The torque arm length may be varied to suit a number of different torque ranges.

Table 1: Instruments used / parameters measured

\begin{tabular}{|l|l|l|l|l|}
\hline Variable & Type & Mfr. & P/N & $\begin{array}{l}\text { Accuracy } \\
(\% \mathrm{FS})\end{array}$ \\
\hline$\omega$ & Encoder & Agilent & HEDS-5540 & $0.32 \%$ \\
\hline$\tau$ & Load Cell & Sensotec & $31 / 1430-04$ & $0.25 \%$ \\
\hline $\mathrm{V}_{\mathrm{s}}$ & V. Divider & & & $1.4 \%$ \\
\hline $\mathrm{I}_{\mathrm{b}}$ & Current Shunt & & & $4 \%$ \\
\hline $\mathrm{I}_{\mathrm{a}}$ & Hall effect & $\begin{array}{l}\text { CR } \\
\text { Magnetics }\end{array}$ & CR5410-30 & $1 \%$ \\
\hline $\mathrm{V}_{\text {motor }}$ & V. Divider & & & $2 \%$ \\
\hline
\end{tabular}

\section{Model Validation}

Figure 6 shows battery voltage during the pronking gait with the results of a Thévenin model overlaid. The source voltage and internal resistance were fit using the MATLAB ${ }^{\mathrm{TM}}$ function polyfit.

Battery voltage during pronking -- actual and predicted based on battery current

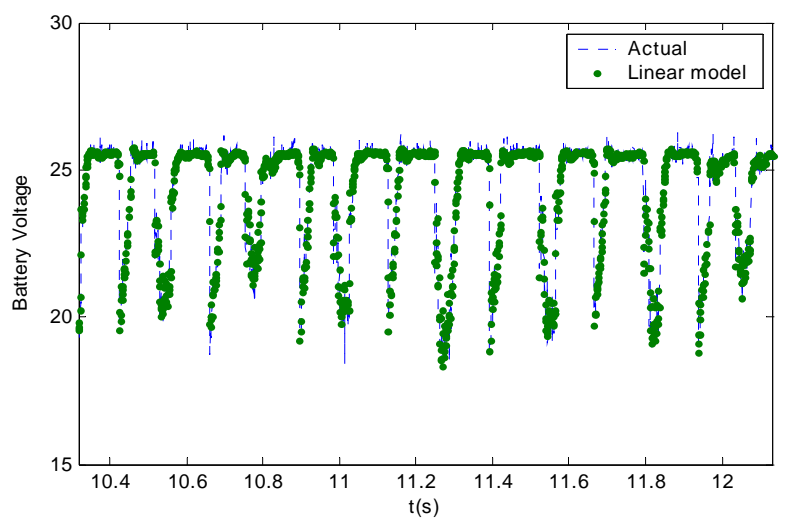

Figure 6: Battery voltage -- actual and predicted based on battery current

The models described above were fit to dynamometer data from a single experimental run. During this trial, load speed was held constant at 5 $\mathrm{rad} / \mathrm{s}$ increments between zero and $40.0 \mathrm{rad} / \mathrm{s}$ by means of a proportional-integral controller. This PI speed controller was not expected to hold the speed exactly constant when DUT torque was high. Instead, speed control was used merely to ensure complete coverage of the first quadrant of the torque speed curve. For each motor speed, commanded duty cycle was driven by a sinusoid command from zero to $100 \%$ and back to zero. Model fits were performed on only the first quadrant of data (positive torques). As the simple models exhibited slightly larger errors in the second quadrant, modeling for this quadrant will be the subject of future work. Figure 7 shows the region of the torque-speed curve swept during the test.

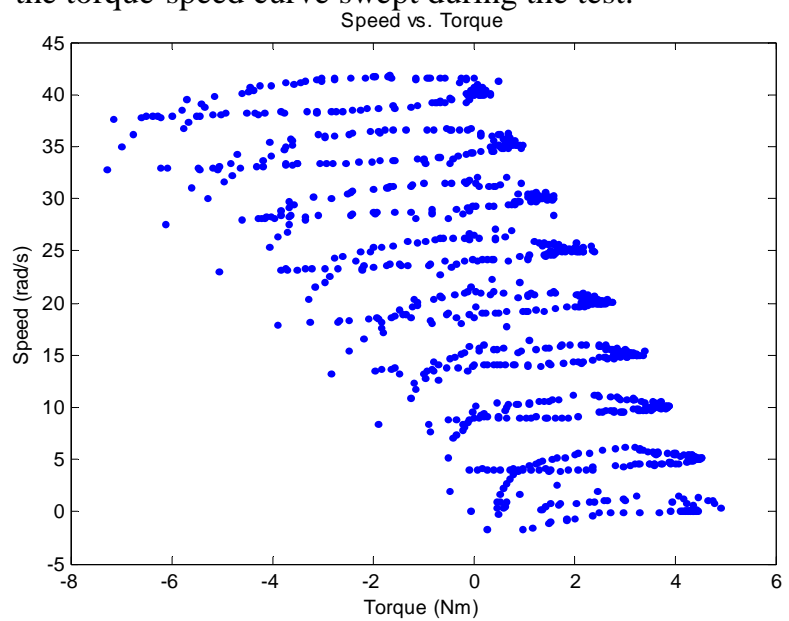

Figure 7: Speed versus torque for the DUT

We begin by examining the input-output relationship of the PWM amplifier described in equation 1. The SA60 amplifier generates a lockedanti-phase PWM signal in response to a command signal in the approximate range $[4.0,8.0] \mathrm{V}$ with the centre roughly at $6.0 \mathrm{~V}$. To determine the exact offset 
voltage and scale factor, as well as to validate the buck converter equation for this circuit, input voltage was swept across its range while measuring output voltage.

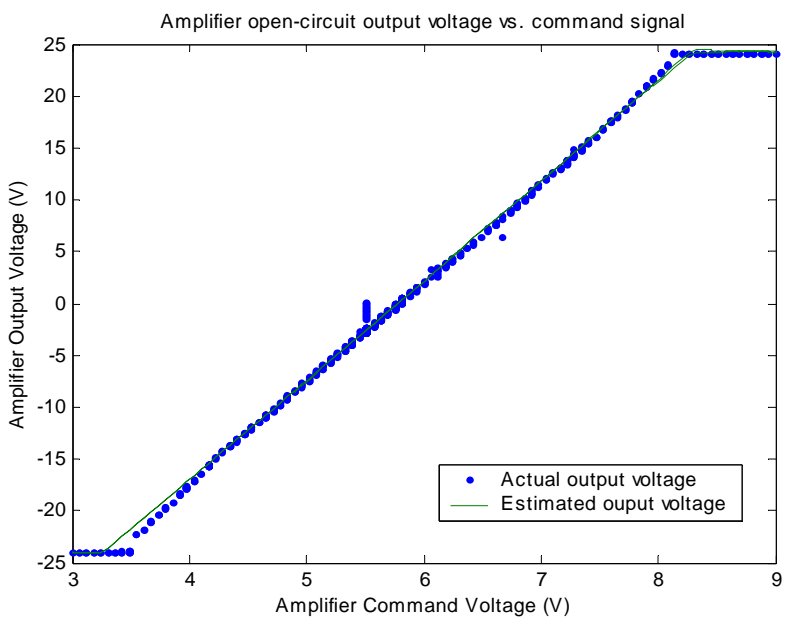

Figure 8: Output of motor amplifier vs. command voltage — actual and estimated

By fitting a line to the linear region of the output voltage versus command signal data using the MATLAB function polyfit, the gain and offset of the motor amplifier were found to be $5.776 \mathrm{~V}$ and 9.565 $\mathrm{V} / \mathrm{V}$ respectively. Figure 8 shows the quality of the fit; the amplifier appears to be linear throughout the middle region while diverging slightly from the model close to $\pm 100 \%$ duty factor.

\section{Terminal voltage during experiment}

Using the above mapping, the remaining parameter of the amplifier model (eq 4a), internal resistance, was determined by fitting estimated terminal voltage during an experiment to actual, using the MATLAB ${ }^{\mathrm{TM}}$ Nelder-Meade minimization program lsqcurvefit. The minimization resulted in a MOSFET internal resistance of $0.444 \mathrm{Ohms}$, close to the Apex SA60 datasheet value of $0.45 \mathrm{Ohms}$ [1].
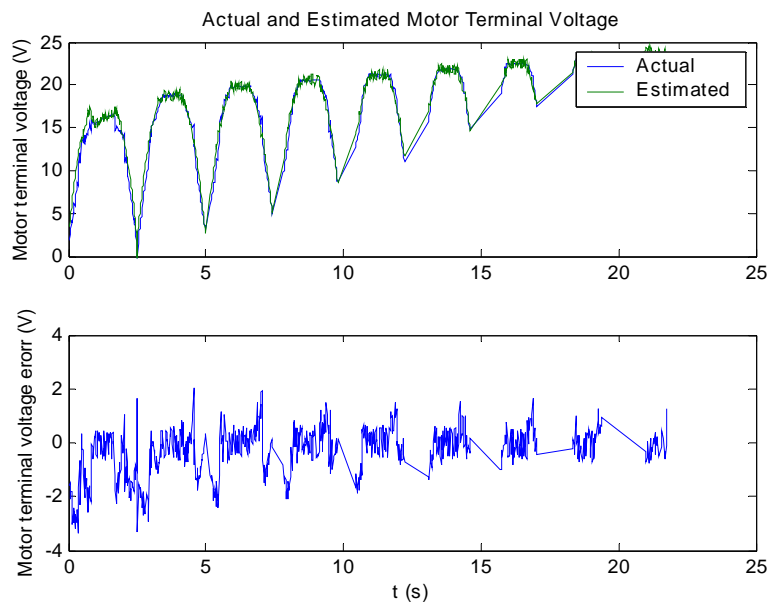

Figure 9: Estimated and actual motor terminal voltage

\section{Motor current estimation}

To calculate the estimated motor current, the output of eq 3 was fit to the real motor current using actual terminal voltage and speed of the motor, and by varying the armature resistance, $\mathrm{R}_{\mathrm{a}}$. A value of $1.65 \Omega$ was found, as compared to the datasheet value of 1.33 $\Omega$.

This model, together with parameters listed below, resulted in the following plot of estimated vs. actual motor current using eq. 5 to determine the estimated motor current.
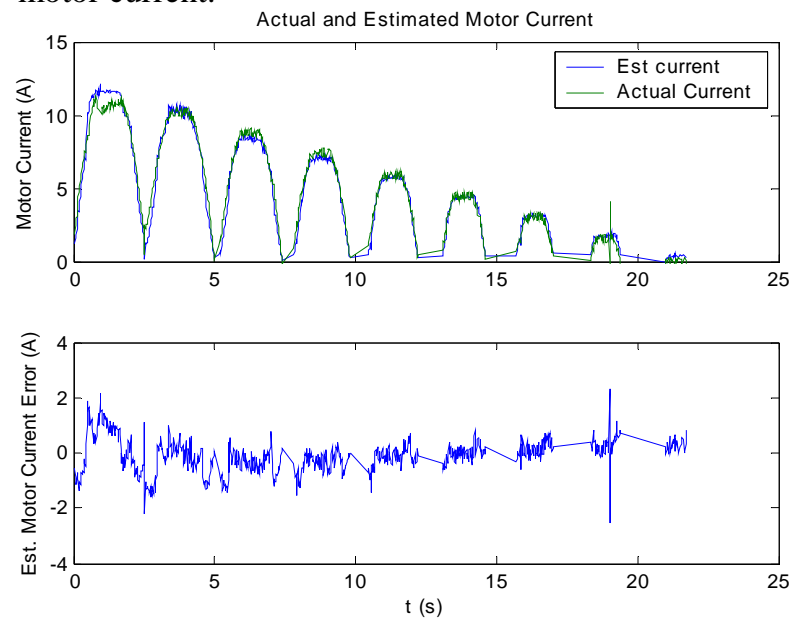

Figure 10: Estimated and actual motor current during experiment

\section{Output Torque}

Output torque was measured with a load cell positioned on a torque arm $87.5 \mathrm{~mm}$ from the axis of the device under test. Using the same process as for the other models, predicted torque was fit to actual torque by adjusting the torque constant, and assuming a gearhead efficiency of $80 \%$. The resulting torque constant, 0.0160 , is close to the datasheet value of 0.0161. Measured torque is compared to that predicted from the measured and estimated motor currents in Figure 11 using the torque constant found.
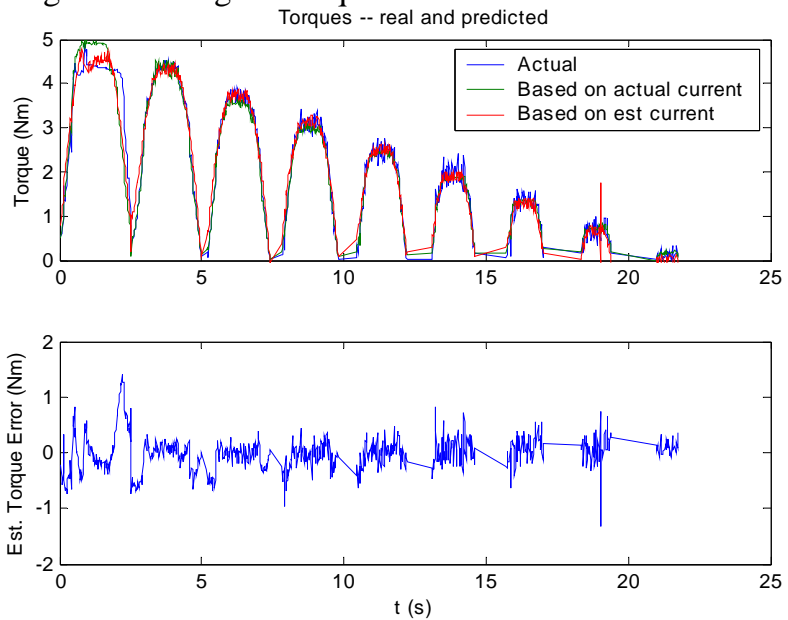

Figure 11: Motor torque-- actual and predicted based on measured and estimated motor current 


\section{Summary of model fit}

To assess the performance of each model, RMS errors between the predicted and actual results for each model were calculated for the first quadrant and for the first and second quadrants together. The results are displayed in Table 2. In particular, in the first quadrant, RMS error for the torque estimate was $5.52 \%$.

Table 2: RMS estimation errors (FS = full-scale)

\begin{tabular}{|c|c|c|c|c|}
\hline \multirow{2}{*}{ Model } & \multicolumn{2}{|l|}{ QI error } & \multicolumn{2}{l|}{ QI, QII error } \\
\cline { 2 - 5 } & $\mathrm{V} / \mathrm{A} / \mathrm{Nm}$ & $\% \mathrm{FS}$ & $\mathrm{V} / \mathrm{A} / \mathrm{Nm}$ & $\% \mathrm{FS}$ \\
\hline $\mathrm{V}_{\text {motor }}=\mathrm{d} \cdot\left(\mathrm{V}_{\mathrm{s}}-\mathrm{d} \cdot \mathrm{i}_{\mathrm{a}} \cdot \mathrm{R}_{\text {amp }}\right)$ & 0.930 & 3.87 & 1.15 & 4.78 \\
\hline $\mathrm{i}_{\mathrm{a}}=\frac{\mathrm{V}_{\text {motor }}-\mathrm{K}_{\mathrm{s}} \omega}{\mathrm{R}_{\mathrm{a}}}$ & 0.606 & 6.06 & 0.680 & 6.80 \\
\hline$\tau=\mathrm{K}_{\mathrm{t}} \frac{\mathrm{d} \cdot \mathrm{V}_{\mathrm{s}}-\mathrm{K}_{\mathrm{s}} \omega}{\mathrm{R}_{\mathrm{a}}+\mathrm{d}^{2} \mathrm{R}_{\text {amp }}}$ & 0.303 & 5.52 & 0.553 & 10.1 \\
\hline
\end{tabular}

Unfortunately, the surprising accuracy of current and torque estimation did not extend to the second quadrant. RMS prediction error in the first and second quadrants together was $10.1 \%$ full-scale, nearly double that of the first quadrant. Although the exact cause of the discrepancy is not yet obvious, Figure 12 shows that the measured torque is consistently larger in magnitude than the estimated torque in the second quadrant. Since second quadrant operation involves substantial current flow through the MOSFET body diodes, it is expected that the circuit model will be slightly different for second quadrant operation.
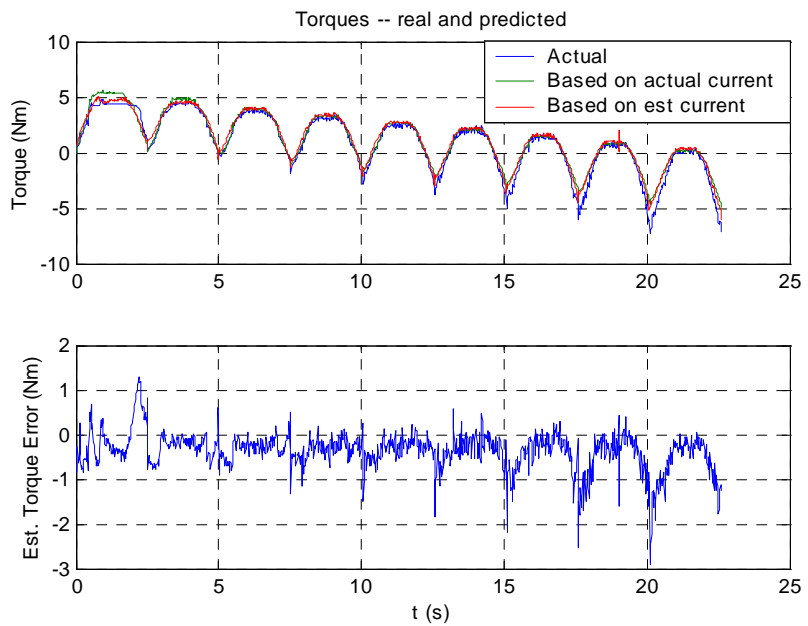

Figure 12: Measured and estimated torques for the first and second quadrants.

\section{Conclusions \& Future Work}

While several issues still remain to be solved, this work has resulted in a motor model capable of predicting output torque within $5.5 \%$ FS during firstquadrant operation, while requiring no sensing at all, besides motor velocity.

Future work will focus both on improving the accuracy of the models presented in this work and on other areas of actuator modeling. For example, a model predicting motor core temperature as a function of case temperature and input current will be sought to help avoid motor failures.

Also, precise measurements of the efficiency of the motor over the operating range may help improve the efficiency of the gaits used on RHex.

\section{Acknowledgements}

This work is supported by DARPA/SPAWAR contract number N66001-00-C-8026. The authors would like to thank members of the RHex team and in particular Uluç Saranli for their contributions.

\section{References}

[1] Apex Microtechology, "SA60 Datasheet" (www.apexmicrotech.com) SA60U Rev. F

[2] Kassakian, J.G., Schlecht, M.F., Verghese, G.C., Principles of Power Electronics, Reading, MA: Addison-Wesley, 1991. p 111.

[3] Maxon Precision Motors, High Precision Drives and Systems (2001 Product Catalog), p 74.

[4] U. Saranli, M. Buehler, and D. E. Koditschek, "RHex: A Simple and Highly Mobile Hexapod Robot," Int. J. Robotics Research, 20(7):616-631, July 2001.

[5] Joel D. Weingarten, Martin Buehler, Richard Groff, Daniel E. Koditschek, "Automated gait generation and optimization for legged robots" IEEE 2003 Int. Conf. Robotics and Automation, Taipei, Taiwan (submitted)

[6] D. Campbell and M. Buehler, "Preliminary Bounding Experiments in a Dynamic Hexapod," In Bruno Siciliano and Paolo Dario, editors, Experimental Robotics VIII, Springer-Verlag, 2002. (in press)

[7] D. McMordie and M. Buehler, "Towards Pronking with a Hexapod Robot", 4th Int. Conf. on Climbing and Walking Robots, Karlsruhe, Germany, September $24-26,2001$.

[8] H. Komsuoglu, D. McMordie, U. Saranli, N. Moore, M. Buehler, and D. E. Koditschek, "Proprioception Based Behavioral Advances in Hexapod Robot", 2001 IEEE Int. Conf. on Robotics and Automation (ICRA), pp 3650 3655, Seoul, Korea, May 21-26, 2001.

[9] D. McMordie, "Towards pronking with a hexapod robot", M. Eng. Thesis, McGill University, 2002.

[10] E.Z. Moore and M. Buehler, "Stable Stair Climbing in a Simple Hexapod", 4th Int. Conf. on Climbing and Walking Robots, Karlsruhe, Germany, September 24-26, 2001.

[11] E. Z. Moore, D. Campbell, F. Grimminger, and M. Buehler, " Reliable Stair Climbing in the Simple Hexapod 'RHex'," 2002 IEEE Int. Conf. on Robotics and Automation (ICRA), Vol 3, pp 2222-2227, Washington, D.C., U.S.A., May 1115,2002

[12] D. Campbell, M. Buehler, "Stair Descent in the Simple Hexpod 'RHex', IEEE 2003 Int. Conf. Robotics and Automation, Taipei, Taiwan (Submitted)

[13] A. E. Fitzgerald, C. Kingsley, Jr., S. D. Umans, Electric Machinery, fifth edition. New York: McGraw-Hill, 1990, p 395. 